Journal of

Molecular Microbiology

and Biotechnology
J Mol Microbiol Biotechnol 2012;22:235-244

DOI: $\underline{10.1159 / 000342449}$
Published online: September 14, 2012

\title{
Staphylococcus aureus Persisters Tolerant to Bactericidal Antibiotics
}

\author{
Sabrina Lechner ${ }^{a}$ Kim Lewis $^{b}$ Ralph Bertram ${ }^{a}$ \\ a Interfakultäres Institut für Mikrobiologie und Infektionsmedizin, Lehrbereich Mikrobielle Genetik, \\ Eberhard Karls Universität Tübingen, Tübingen, Germany; ${ }^{\mathrm{b}}$ Antimicrobial Discovery Center, Department of Biology, \\ Northeastern University, Boston, Mass., USA
}

\section{Key Words}

Persister cells · Multidrug tolerance $\cdot$ Antibiotics •

Antimicrobials $\cdot$ Staphylococcus aureus

\begin{abstract}
Bacterial persister cells are non- or slow-growing reversible phenotypic variants of the wild type, tolerant to bactericidal antibiotics. We analyzed here Staphylococcus aureus persister levels by monitoring colony-forming unit counts of planktonically grown cells treated with six different antimicrobials over time. The model laboratory strains HG001-HG003, SA113 and the small colony variant (SCV) strains hemB and men $D$ were challenged by the compounds at different logs of minimal inhibitory concentration (MIC) in exponential or stationary growth phase. Antibiotic tolerance was usually elevated in SCV strains compared to normally growing cells and in stationary versus exponential phase cultures. Biphasic killing kinetics, typical for persister cell enrichment, were observed in both growth phases under different selective conditions. Treatment of exponential phase cultures of HG001HG003 with 10-fold MIC of tobramycin resulted in the isolation of persisters which upon cultivation on plates formed either normal or phenotypically stable small colonies. Trajectories of different killing curves indicated physiological heterogeneity within persister subpopulations. Daptomycin added at 100 -fold MIC to stationary phase SA113 cells rap-
\end{abstract}

idly isolated very robust persisters. Fractions of antibiotictolerant cells were observed with all S. aureus strains and mutants tested. Our results refute the hypothesis that $S$. aureus stationary phase cells are equivalent to persisters, as not all of these cells showed antibiotic tolerance. Isolation of S. aureus persisters of different robustness seems to depend on the kind and concentration of the antibiotic, as well as on the strain used.

Copyright $\odot 2012$ S. Karger AG, Basel

\section{Introduction}

Bacterial persister cells were described by Joseph W. Bigger [1944] who studied the action of penicillin against staphylococci. Addition of the antibiotic to liquid cultures led to the sorting out of a small number of survivors he termed persisters. Upon reinoculation, these cells gave rise to a culture that again was susceptible to the antibiotic, forming a new subpopulation of persisters. Decades later, comparable observations were made [Gunnison et al., 1964; Mayhall et al., 1976; Newsom, 1970], confirming that a fraction of Staphylococcus aureus cells within a common culture survived antibiotic treatment although the original strains were susceptible to it. These cells were neither spheroplasts nor L-forms, they did not arise in response to the antibiotic and tolerance was not trans-

\section{KARGER \\ Fax +41613061234 E-Mail karger@karger.ch} www.karger.com
(C) 2012 S. Karger AG, Basel $1464-1801 / 12 / 0224-0235 \$ 38.00 / 0$

Accessible online at: www.karger.com $/ \mathrm{mmb}$
Ralph Bertram

Interfakultäres Institut für Mikrobiologie und Infektionsmedizin

Lehrbereich Mikrobielle Genetik, Eberhard Karls Universität Tübingen

Waldhäuser Strasse 70/8, DE-72076 Tübingen (Germany)

E-Mail ralph.bertram@uni-tuebingen.de 
ferred to the progeny. Recently staphylococcal persisters were revisited, providing insights into the distribution of S. aureus and S. epidermidis persisters in biofilms and planktonically grown cells [Keren et al., 2004a; Möker et al., 2010; Shapiro et al., 2011]. Persisters have been identified in an increasing number of microorganisms, including Pseudomonas aeruginosa [Brooun et al., 2000; Harrison et al., 2005; Möker et al., 2010; Spoering and Lewis, 2001], Escherichia coli [Keren et al., 2004a, b; Shah et al., 2006], Mycobacterium tuberculosis [Keren et al., 2011] and the unicellular fungus Candida albicans [Lafleur et al., 2010; reviewed in Lewis, 2010a]. To identify genetic mechanisms underlying the persister state, Moyed and colleagues [Moyed and Bertrand, 1983; Moyed and Broderick, 1986; Scherrer and Moyed, 1988] carried out a selection for E. coli mutants with increased survival to ampicillin, which resulted in the identification of a mutant carrying the hipA7 allele producing elevated amounts of persisters. Persisters are presumably formed due to stochastic variation in the levels of persister gene expression irrespective of antibiotic action [Balaban et al., 2004]. At the same time, the probability of persister formation can be strongly increased by stress. It was reported that the majority of $E$. coli persisters under DNA-damaging conditions are produced by expression of the TisB toxin, which is under the control of the SOS response [Dörr et al., 2009]. By definition, persisters are not mutants, but are regarded as phenotypic variants that are isogenic to normally growing cells within a common culture [Lewis, 2007, 2010a, b; Wiuff et al., 2005]. Persister levels in most species examined increase sharply during late exponential growth, reaching $\sim 1 \%$ at the stationary phase [Keren et al., 2004a]. Unraveling the molecular mechanisms of persister formation in species other than E. coli has only recently begun, reflected by recent studies in $P$. aeruginosa or M. tuberculosis [Keren et al., 2011; Möker et al., 2010]. Regarding S. aureus, the observation that a stationary culture of strain ATCC 55585 was completely tolerant to the fluoroquinolone ciprofloxacin had suggested that stationary cells may be equivalent to persisters in this organism [Keren et al., 2004a]. We examined this possibility in the present study and found that stationary cultures of $S$. aureus, similarly to other species examined, represent a mixture of regular cells and persisters. We also considered persister formation by $S$. aureus small colony variant (SCV) strains that are intrinsically tolerant to selected antibiotics. SCVs are often isolated in hospitals and contribute to antibiotic recalcitrance of infections [von Eiff, 2008]. SCVs from both clinical isolates and genetically manipulated mutants are frequently impaired in the respiratory chain. They may carry mutations in hemB that is involved in hemin biosynthesis [von Eiff et al., 1997], or menD required for menaquinone production [Bates et al., 2003], or may be auxotrophic for thymidine. Notably, their smaller size pertains to the colony but not on the single-cell level [Proctor et al., 2006]. Whether $\mathrm{SCV}$ are also affected in persister production is an important question which we examined in this study.

\section{Results}

Examination of Persister Levels of Exponential Phase S. aureus Cells

To isolate S. aureus persister cells, we wanted to identify conditions under which (1) the bulk of cells of a culture is rapidly eradicated after the addition of an antimicrobial compound; (2) a low percentage of cells within this culture is still capable of forming colonies on nonselective rich media after up to $48 \mathrm{~h}$, and (3) the colonyforming unit (CFU) count of the liquid culture does not increase to a sizeable extent after the antibiotic challenge, but exhibits a plateau or a slow decrease during the course of the experiment. Such outcomes are reflected by biphasic killing curves when CFU counts are plotted against time [Balaban et al., 2004; Dhar and McKinney, 2007]. During extensive in vitro antibiotic challenge experiments, we tested model laboratory $S$. aureus strains (table 1) exhibiting either normal growth or an SCV phenotype in liquid rich medium by five different antibiotics and one disinfectant applied at 1-, 10-, and 100-fold the minimal inhibitory concentration (MIC). These compounds and respective MIC values determined at the onset of this study are summarized in table 2. In a first approach, time-dependent eradication of exponentially grown cells was monitored. Results explicated in the following are restricted to conditions under which the trajectories of resulting killing curves indicated either persister isolation or other interesting phenomena. When ciprofloxacin was added to the cultures at 10-fold MIC, a rapid decrease in CFU counts was observed ranging from $94 \%$ (menD) to $99 \%$ (SA113) reduction after $2 \mathrm{~h}$, followed by rather stable values for up to $24 \mathrm{~h}$, indicating the presence of ciprofloxacin-tolerant persisters (fig. 1a). SCV strains were more tolerant to the drug than strains HG001, HG002, and HG003 (in the following referred to as 'HG strains' due to mostly uniform behavior in this study) and SA113, which were killed by ciprofloxacin at comparable rates. In the presence of 100-fold MIC of rifampin, live counts of SA113 decreased drastically, 
Table 1. Strains used in this study

\begin{tabular}{lll}
\hline S. aureus strains & Relevant characteristic(s) & Reference \\
\hline HG001 & NCTC8325 derivative, $r s b U$ repaired & Herbert et al., 2010 \\
HG002 & NCTC8325 derivative, $t$ caR repaired & Herbert et al., 2010 \\
HG003 & NCTC8325 derivative, $r$ sbU and $t c a R$ repaired & Herbert et al., 2010 \\
SA113 (ATCC 35556) & NCTC8325 derivative, $a g r^{-}, 11$-bp deletion in $r s b U$ & Iordanescu and Surdeanu, 1976 \\
SA113 hemB & SA113 hemB:ermB, SCV phenotype & Gaupp et al., 2010 \\
SA113 menD & SA113 menD:erm , SCV phenotype & Gaupp et al., 2010 \\
\hline
\end{tabular}

Table 2. MIC values of antimicrobial agents $(\mu \mathrm{g} / \mathrm{ml})$

\begin{tabular}{|c|c|c|c|c|c|c|c|}
\hline Strain & Penicillin G & Ciprofloxacin & Rifampin & Tobramycin & Daptomycin & $\begin{array}{l}\text { Daptomycin + } \\
\mathrm{Ca}^{2+}\end{array}$ & BAC \\
\hline HG001, HG002, and HG003 & 0.02 & 0.31 & 0.01 & 1.25 & 50 & 4.0 & 1.6 \\
\hline SA113 & 0.02 & 0.3 & 0.02 & 0.6 & 25 & 4.0 & 1.6 \\
\hline SA113 hemB & 0.01 & 0.2 & 0.02 & 31 & 25 & 6.5 & 1.4 \\
\hline SA113 menD & 0.01 & 0.2 & 0.02 & 31 & 25 & 6.5 & 1.4 \\
\hline
\end{tabular}

$\mathrm{BAC}=$ Benzalkonium chloride.

whereas killing of HG strains and particularly SCV strains was considerably slower during the first $3 \mathrm{~h}$ (fig. 1b). When cultures were challenged by 10 -fold MIC of tobramycin, live counts of all strains decreased by three or four orders of magnitude during the 1st hour and leveled off within the next $7 \mathrm{~h}$ in non-SCV strains (fig. 1c). Colonies grown from HG cells surviving this treatment gave rise to two different morphotypes. About $78 \%$ of them had the size of the parent strains, whereas $\sim 22 \%$ had a stable, non-reverting SCV appearance. Treatment with tobramycin at 100-fold of the MIC resulted in rapid killing of the entire hemB and menD population, whereas CFU values of SA113 decreased by about four orders of magnitude after $1 \mathrm{~h}$, followed by a rather slow decrease during the next $5 \mathrm{~h}$ (fig. 1d). Inexplicable fluctuations in case of HG strains precluded unequivocal conclusions under these conditions. Treatment of hemB and menD with 10-fold MIC of daptomycin resulted in a slightly biphasic killing pattern (fig. 1e), whereas the majority of SA113 cells were killed within the 1st hour and the remainder was eradicated completely after $4 \mathrm{~h}$. Again, respective values were less meaningful with HG strains. The activity of daptomycin is strongly increased in the presence of calcium ions [Steenbergen et al., 2005], and indeed applying 100- or 10-fold the MIC of daptomycin with $50 \mu \mathrm{g} / \mathrm{ml} \mathrm{Ca}^{2+}$ eradicated all cultures within up to 4 or $5 \mathrm{~h}$, respectively. The complete set of killing curves with exponential-phase $S$. aureus cultures, including treatments with additional antimicrobials listed in table 2 , is provided in online supplementary figure $1 \mathrm{~A}-\mathrm{G}$ (for all online supplementary material, see www. karger.com/doi/10.1159/000342449).

\section{Isolation of Persister Cells in Stationary Phase}

\section{S. aureus Cultures}

In order to reappraise whether all stationary growth phase $S$. aureus cells actually represent persisters, overnight cultures were treated with the same set of antimicrobials (except penicillin G) applied at identical concentrations as before. In contrast to treatment with higher concentrations of the quaternary ammonium compound benzalkonium chloride, which resulted in the complete eradication of all strains tested within $3 \mathrm{~h}$ or less (online suppl. fig. 1G), a high degree of drug tolerance irrespective of strains or antibiotic concentration was observed in most cases. Notably, tobramycin or daptomycin challenge resulted in selective eradication of cells. CFU values of most strains declined slowly upon treatment with 100fold MIC of tobramycin whereas live counts of menD cells decreased by $94.6 \%$ within the 1 st hour or by $99.9 \%$ with- 

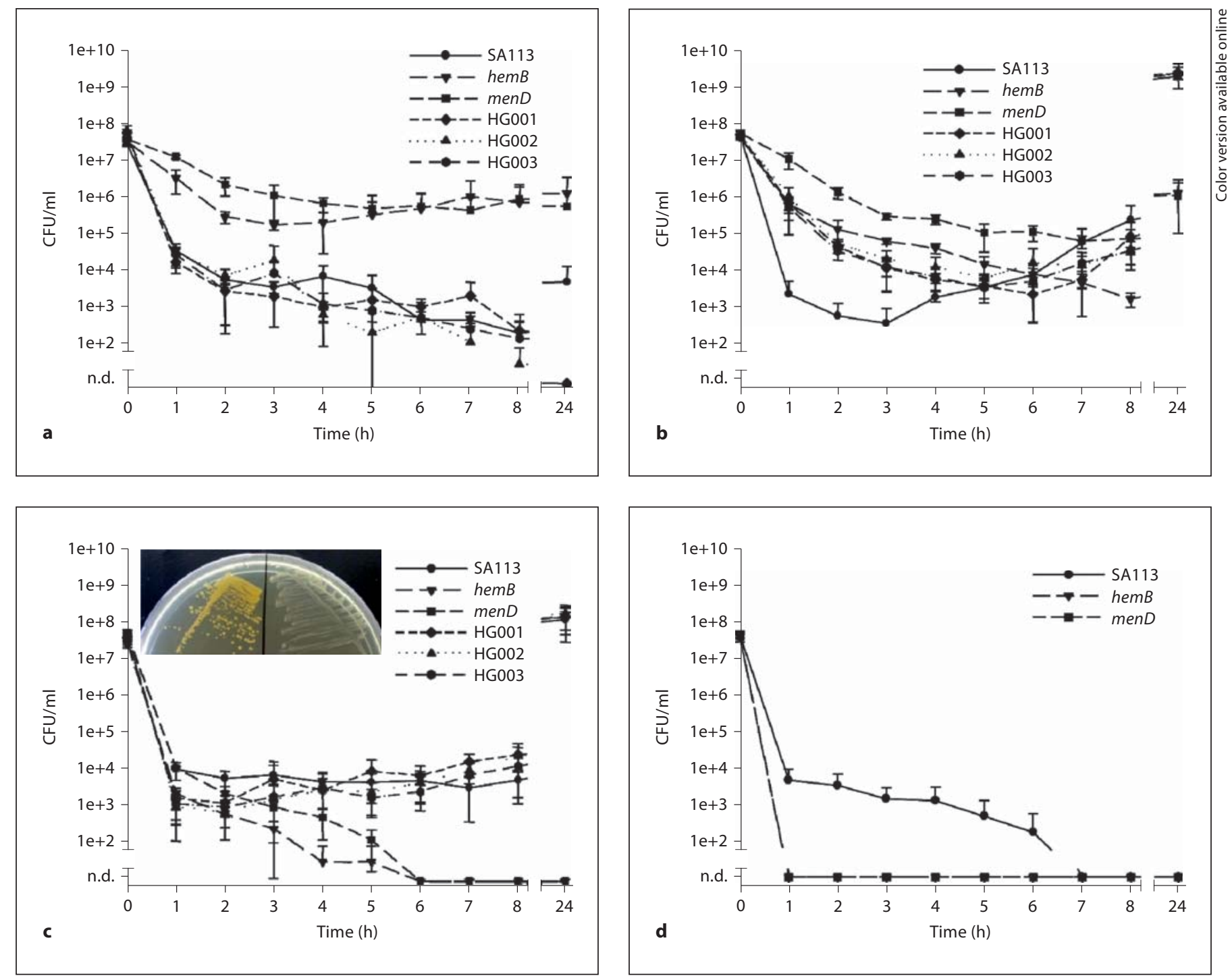

Fig. 1. Time-dependent killing of exponential phase cultures. Strains were treated with 10-fold MIC of ciprofloxacin (a), 100fold MIC of rifampin (b), 10-fold MIC of tobramycin (c), 100-fold MIC of tobramycin (d), or 10-fold MIC of daptomycin (e). The limit of detection was $100 \mathrm{CFU} / \mathrm{ml}$ throughout all killing experiments. Error bars indicate standard deviations. The plate segment (c) shows colonies of untreated HG001 cells (left) and SCV colonies obtained from the HG001 strain $1 \mathrm{~h}$ after tobramycin treatment (right). Note also the loss of pigmentation, typical for SCV strains. 


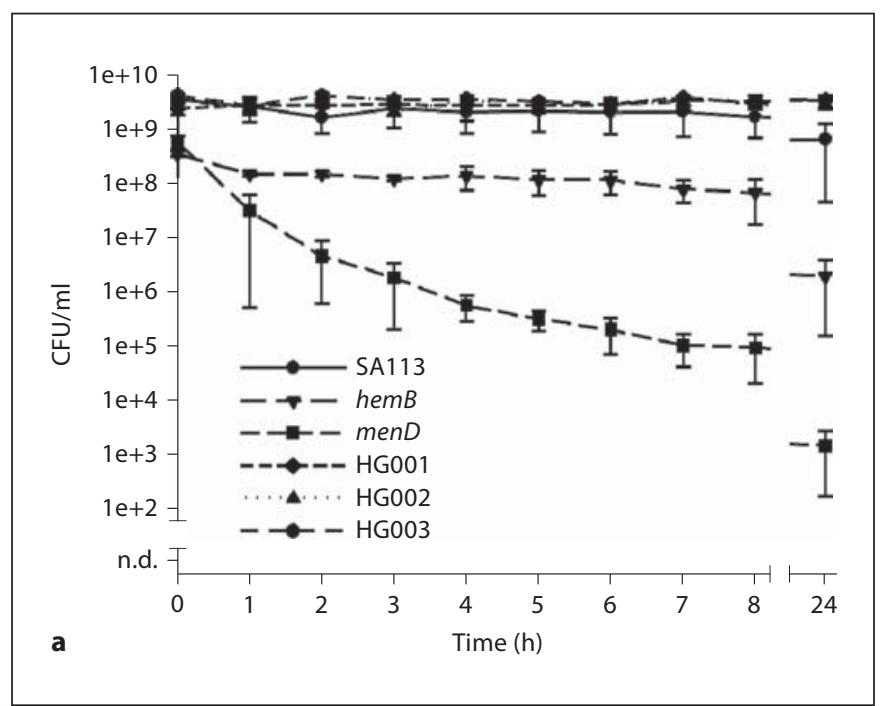

Fig. 2. Time-dependent killing of stationary phase cultures. Strains were treated with 100-fold MIC of tobramycin (a), 100 fold MIC of daptomycin (b), or 100-fold MIC of daptomycin/Ca ${ }^{2+}$ (c).

in $4 \mathrm{~h}$, respectively, followed by an $84 \%$ reduction between time points $\mathrm{t}=4 \mathrm{~h}$ and $\mathrm{t}=8 \mathrm{~h}$ (fig. $2 \mathrm{a}$ ). Challenging SA113 with 100-fold MIC of daptomycin yielded a killing curve that obeyed a very distinct biphasic pattern (fig. 2b). The bulk of cells (99.98\%) were eradicated within $1 \mathrm{~h}$, followed by a subsequent CFU decrease by only about $13 \% / \mathrm{h}$ for the next $7 \mathrm{~h}$ or by about $4.3 \% / \mathrm{h}$ for the next $23 \mathrm{~h}$ on average, respectively. Of note, killing curves observed with 100 -fold MIC of daptomycin/ $/ \mathrm{Ca}^{2+}$ were biphasic not only in case of SA113 but clearly also for the menD strain which had been unaffected by daptomycin alone (fig. 2c). CFU counts of HG strains declined rather continuously whereas the hemB population remained relatively stable. Addition of $1 \mu \mathrm{g} / \mathrm{ml}$ hemin to the hemB culture resulted


in killing kinetics almost indistinguishable to those of SA113 (online suppl. fig. 2). The complete set of killing curves observed with stationary phase $S$. aureus cultures can be found in online supplementary figure 3 .

\section{Stationary Phase SA113 Cells Are Rapidly Eradicated}

by Treatment with Combined Super-MICs of

Daptomycin and Tobramycin

An overnight culture of SA113 was challenged simultaneously with 100-fold MIC each of daptomycin and tobramycin, to combine both conditions which had resulted in pronounced isolation of persisters before. Killing kinetics observed were clearly different compared to those of cultures treated with either of the drugs alone 


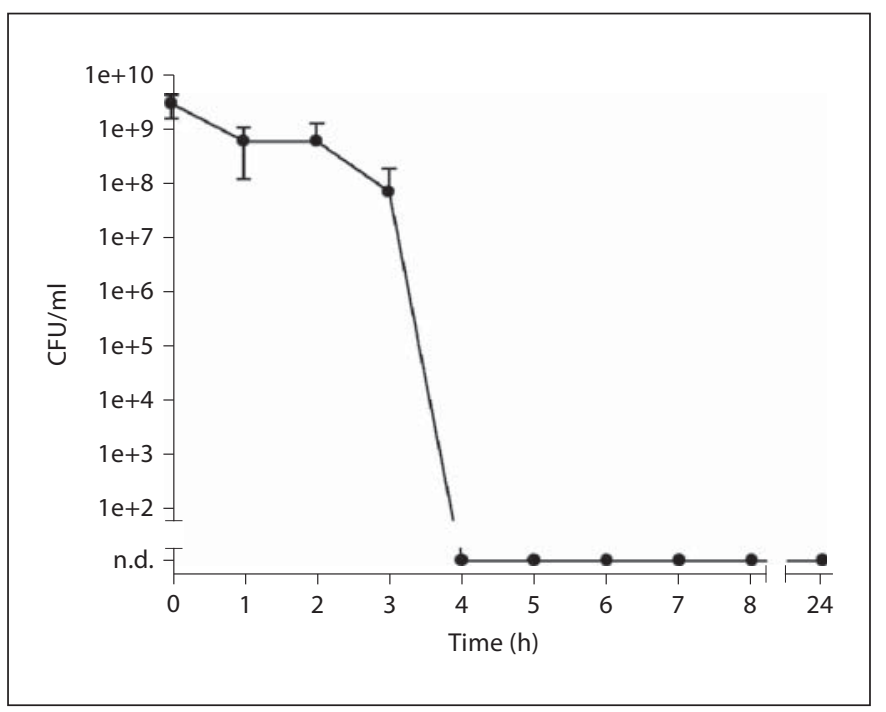

Fig. 3. Time-dependent killing of stationary phase SA113 by combined daptomycin and tobramycin treatment. Both antibiotics were applied at 100 -fold MIC.

(fig. 3). After a 97\% decline in CFU values during the first $3 \mathrm{~h}$, the remaining population was entirely eradicated between time points $\mathrm{t}=3 \mathrm{~h}$ and $\mathrm{t}=4 \mathrm{~h}$, which indicates a synergistic effect in antibacterial activity exerted by these two antibiotics.

\section{Long-Term Antibiotic Challenge Leads to Complete Eradication of SA113 Cells}

In order to assess the stability of persister levels over longer periods of time, CFU values of cultures treated with 100-fold MICs of antibiotics for 21 days were monitored. All cultures underwent considerable decreases in live counts within the first 7 days or earlier, whereas untreated cultures maintained high CFU levels during the entire course of the experiment. Formation of colonies on nonselective plates ceased, when cultures had been challenged with daptomycin or tobramycin for 3 days, with rifampin for 7 days or with ciprofloxacin for 21 days (fig. 4). The recurring CFU increase of cultures in the presence of penicillin $\mathrm{G}$ is rationalized by a concomitant constant decrease in antimicrobial activity within up to 10 days, whereas all other compounds tested were active up to the time of complete persister eradication or beyond (online suppl. fig. 4).

\section{The Persister State Is Not Inherited in SA113 Cells}

Exponential cultures of SA113 were repeatedly sorted out for persisters by treatment with 100 -fold MIC of tobra- mycin or 10-fold MIC of daptomycin to reveal whether antibiotic tolerance of $S$. aureus persisters is inherited over many generations. As depicted in figure $5 \mathrm{a}$ and $\mathrm{b}$, respective killing curves after up to four cycles still resembled those of the original populations. The fact that persister levels were not elevated in later cycles indicates that the persister state of $S$. aureus is not vertically transmitted.

\section{Discussion}

Although staphylococcal persisters had been described more than 60 years ago [Bigger, 1944], a profound analysis of this drug-tolerant and putatively dormant state for this genus is lacking. We hence addressed a number of different questions regarding $S$. aureus persisters: (1) Do all stationary phase cells of $S$. aureus reside in the persister state? (2) Do exponential phase cultures also contain persister cells and, if so, to what extent? (3) Is there a correlation between persister levels and strain and genotype backgrounds? (4) Does the kind and concentration of an antimicrobial agent applied to comparable cultures influence persister levels? To establish a method for isolating drug-tolerant $S$. aureus persisters, antibiotics were applied which act on most of the common bacterial target structures, such as the cell envelope (penicillin, daptomycin), ribosome (tobramycin), RNA polymerase (rifampin), or topoisomerase (ciprofloxacin). Sorting for persisters was observed independently of the chemical nature or the target of the drug in accordance with previous studies on S. epidermidis [Shapiro et al., 2011] and other bacteria [Keren et al., 2011; Spoering and Lewis, 2001]. Generally, S. aureus cells in stationary growth phase were less vulnerable by antibiotics than those in exponential phase, arguably due to elevated persister levels [Wiuff et al., 2005]. Under most of the conditions we tested, the survival rates of stationary phase cells were either unaffected by antibiotic treatment (particularly upon challenge with 1- or 10-fold MICs of drugs) or CFU counts decreased rather constantly during $24 \mathrm{~h}$ (see online suppl. fig. 3). By contrast, the strong recurring increase in CFU counts of exponential phase SA113 treated with 100-fold MIC rifampin (fig. 1b) indicated genetically determined changes in susceptibility. These can be achieved by single amino acid exchanges in RNA polymerase $\beta$-subunit (RpoB) of $S$. aureus [Aubry-Damon et al., 1998]. Indeed, rifampin-challenged cells forming colonies on nonselective plates also grew well when restreaked on rifampin-supplemented plates (data not shown). During the antibiotic challenge procedure, a 
Fig. 4. Long-term killing experiment. Stationary phase SA113 cultures were treated with 100-fold MIC each of daptomycin, tobramycin, ciprofloxacin, rifampin, or penicillin for 21 days. An identical SA113 culture without any antibiotic challenge served as a control.
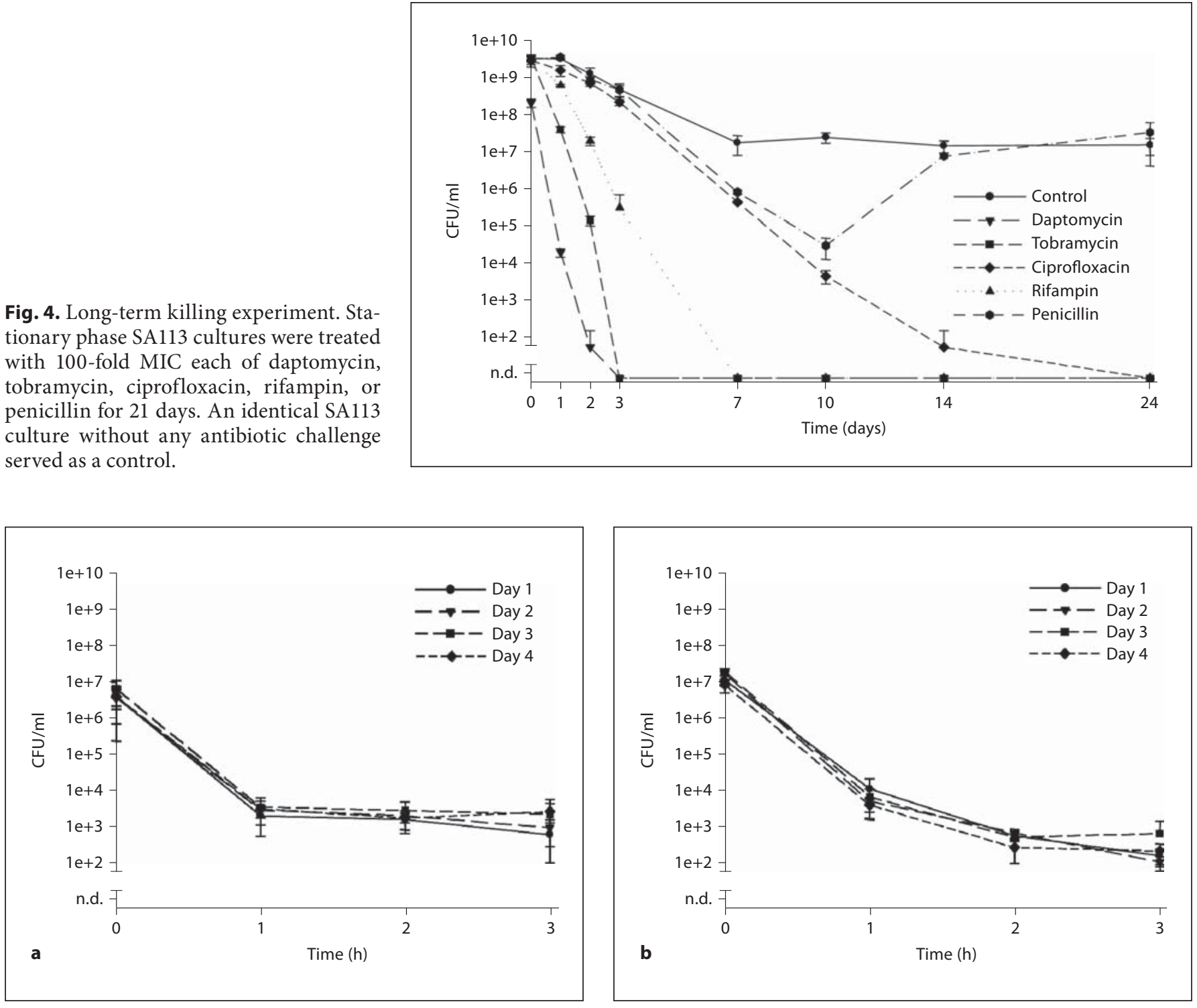

Fig. 5. Test for heritability of persistence. Exponential phase SA113 cells were challenged with 100-fold MIC of tobramycin (a) or 10fold MIC of daptomycin/ $\mathrm{Ca}^{2+}(\mathbf{b})$ for $3 \mathrm{~h}$ in four consecutive cycles.

higher selective pressure for drug resistance was presumably exerted on populations mainly composed of rapidly killed cells. Accordingly, recovery of CFU counts was not observed with the more tolerant hemB and menD strains within $8 \mathrm{~h}$. Eradication of exponential phase cells was most rapidly achieved with 10- and 100-fold MICs of the cyclic lipopeptide daptomycin, and this effect was augmented by the addition of $\mathrm{Ca}^{2+}$ cations [Steenbergen et al., 2005]. Indeed, daptomycin/ $\mathrm{Ca}^{2+}$ is effective against a number of $S$. aureus strains including hemB [Baltch et al., 2008; Begic et al., 2009; Mascio et al., 2007], and killing kinetics of S. aureus ATCC 29213 cultures challenged with super-MICs of daptomycin/ $\mathrm{Ca}^{2+}$ had previously also displayed biphasic trajectories [Mascio et al., 2007], albeit not as pronounced as in the present study. Treatment of HG cells with 10 -fold MIC of tobramycin in exponential growth phase isolated persisters that gave rise to both normally growing and small-colony variants in agreement with previous studies using the related aminoglycoside compound amikacin against $S$. aureus [Singh et al., 2009]. Tiny staphylococcal colonies had also been discovered by Bradley et al. [1978] when clinical isolates were 
tested for tolerance towards oxacillin, cephalothin, or gentamicin. These had resumed the same size, pigmentation, and hemolytic characteristics as the parent strain after $24 \mathrm{~h}$, which is in contrast to the phenotypically stable SCVs we obtained (fig. 1c). Whether the latter are the result of nongenetic heterogeneity [Tuchscherr et al., 2011] or of mutations [von Eiff et al., 1997; Wiuff et al., 2005] may be clarified by analyzing possible hemin, thymidine, thiamine, or menadione auxotrophies due to defects in hemB, menD, or other factors.

Persisters of $S$. aureus, like other bacteria, appear to be physiologically heterogeneous in terms of robustness or fitness [Allison et al., 2011a]. Exposure of identical S. aureus cultures, assumed to contain a reproducible mixture of cells in different physiologic states, to different antibiotics resulted in a variety of killing curves. Some of these exhibited pronounced biphasic trajectories, indicating that the respective drug rapidly sorts for highly robust persisters (e.g. SA113 treated with 100 -fold daptomycin in fig. $2 b$ ), whereas curves with rather smooth transitions from rapid to slower killing speak in favor of a compound which can be endured for longer periods also by less tolerant persisters. This is exemplified by menD cultures challenged in the stationary growth phase by tobramycin (fig. 2a), which kills nongrowing cells by interrupting translation. Resulting misfolded toxic peptides [Correia et al., 2006] are assumed to influence the persister state [Vazquez-Laslop et al., 2006].

As expected, not even very robust $S$. aureus persisters seem to be indefinitely inert against antimicrobials. During long-term experiments persisters of SA113 were eradicated completely when exposed to active drugs at high concentrations within 3-21 days (fig. 4; online suppl. fig. 4). Simultaneous treatment of stationary phase SA113 cultures with high concentrations of daptomycin and tobramycin eradicated persister cells completely within few hours, revealing a synergistic bactericidal effect exerted by this pair of drugs. It is conceivable that passive tobramycin entrance into cells was facilitated by daptomycinmediated membrane damage, similar to the activated uptake of aminoglycosides into persister cells upon generation of a proton motive [Allison et al., 2011b]. In line with our results, Baltch et al. [2008] observed more efficient killing of $S$. aureus strains SH1000 and Newman upon treatment with a combination of daptomycin $/ \mathrm{Ca}^{2+}$ and gentamicin than with either of the drugs alone. These results support the strategy to combat bacterial infections by applying suitable combinations of drugs [Cottarel and Wierzbowski, 2007]. It should be noted, though, that our study is generally restricted to provide model conditions for isolating persisters, because conditions of 100 -fold the MIC of antibiotics can hardly be achieved during clinical treatment.

The use of different well-established laboratory S. aureus strains and mutants demonstrates the genetic and genomic influence on bacterial persister levels. Generally, among the strains tested, SA113 was most susceptible to the applied antimicrobials, which may in part be due to its short generation time, particularly compared to SCV strains. Except for chromosomal resistance cassettes inactivating hemB or menD, SCVs of this study are isogenic to SA113 [Gaupp et al., 2010] and indeed chemical complementation of a hemB mutant resulted in an eradication pattern almost identical to SA113 (as tested with 100-fold $\mathrm{MIC}$ of daptomycin $/ \mathrm{Ca}^{2+}$, see online suppl. fig. 2). By contrast, genomic comparison between HG strains and SA113, all derived from isolate RN1 [Novick, 1967], is less straightforward. SA113 was obtained by unspecific chemical mutagenesis and its genome sequence is unavailable, whereas HG strains resulted from targeted repairs of the transcriptional regulators RsbU and TcaR [Herbert et al., 2010]. The generally homogenous killing curves among HG strains indicate that neither of these two factors affects $S$. aureus persister levels. This is particularly remarkable in case of RsbU, which positively regulates the alternative transcription factor $\sigma^{\mathrm{B}}$ [Giachino et al., 2001], influences the expression of the mazEF toxin-antitoxin system [Donegan and Cheung, 2009; Senn et al., 2005], and downregulates the agr locus [Bischoff et al., 2001] encoding quorum sensing factors [Novick and Geisinger, 2008]. Overexpression of the MazF toxin has been described to induce bacteriostasis in $S$. aureus [Fu et al., 2009], which may have implications for persister formation. An interesting new finding points towards indoleinduced persister formation in E. coli [Vega et al., 2012] and studies by Shapiro et al. [2011] showed that quorum sensing does not alter persister levels in S. epidermidis. Further studies will clarify whether small molecules influence persister levels in S. aureus.

\section{Conclusions}

We here demonstrate that growth phase, strain background, and genotype influence $S$. aureus persister levels. As in other bacteria, the concentration of these drug-tolerant cells clearly increased from exponential to stationary phase cultures and the persister state does not seem to be inherited [Keren et al., 2004a]. A systematic analysis of persister formation with clinical S. aureus isolates and 
mutants is now required and so are clinically applicable strategies to kill S. aureus persisters. Recently, interesting approaches exploiting compounds specifically targeting persisters or the generation of proton motive force in persisters have been described [Allison et al., 2011b; Kim et al., 2011]. In the future, we need to learn more about the distribution of drug tolerant $S$. aureus strains and the genetic mechanisms pertaining to this phenomenon in order to catch up with the state of knowledge regarding genetically drug resistant $S$. aureus strains and lineages [Chambers and Deleo, 2009].

\section{Methods}

Bacterial Strains, Media, and Culture Conditions

$S$. aureus strains used in this study are listed in table 1 . Unless stated otherwise, bacteria were cultured at $37^{\circ} \mathrm{C}$ with aeration in baffle flasks containing tryptic soy broth (TSB; Sigma) at a 1:6 culture-to-flask ratio or on tryptic soy agar (TSA). Liquid cultures were shaken at $150 \mathrm{rpm}$. To chemically complement the SCV phenotype of the SA113 hemB mutant, hemin (Sigma) was added to a final concentration of $1 \mu \mathrm{g} / \mathrm{ml}$ where indicated.

\section{Reagents}

Daptomycin analytic grade powder (designated 'Cubicin') was purchased from Novartis Pharma. Facultatively, $\mathrm{Ca}^{2+}$ cations (50 $\mu \mathrm{g} / \mathrm{ml}$ final concentration), provided as $\mathrm{CaCl}_{2}$, were added to daptomycin-treated cultures, to increase antibiotic activity where indicated. Ciprofloxacin was obtained from Fluka. Tobramycin, penicillin G, rifampin, and benzalkonium chloride were purchased from Sigma. Solutions of antimicrobials were prepared freshly prior to each application and were sterilized using a filter of $0.2-\mu \mathrm{m}$ pore size (Whatman).

\section{Susceptibility Testing}

The MIC of each antimicrobial compound was determined in triplicate by a conventional broth microdilution technique in TSB. The MIC was defined as the lowest antibiotic concentration that inhibited visible bacterial growth (according to $\mathrm{OD}_{578}$ measurements) after $24 \mathrm{~h}$ of incubation at $37^{\circ} \mathrm{C}$.
Time-Kill Studies of Exponential and Stationary Phase

Staphylococci Challenged with Antimicrobials

To determine the number of persister cells in exponential phase, cells were grown overnight in 8 or $16 \mathrm{ml}$ TSB and were inoculated to $16 \mathrm{ml}$ of fresh media to an initial $\mathrm{OD}_{578}$ of 0.07 . Cultures were shaken for 1.5-2 h (for normally growing cells) or 2.5$3 \mathrm{~h}$ (for SCVs), until an $\mathrm{OD}_{578}$ of approximately 0.5 was reached. To determine the number of persister cells in stationary phase, overnight cultures were used. In both cases, $2.24 \mathrm{ml}$ of the overnight or the exponential phase culture was transferred to $14-\mathrm{ml}$ culture tubes (Greiner), antimicrobials were added at 1- to 100fold MIC as indicated and cultures were shaken for $24 \mathrm{~h}$ or for 21 days during long-term experiments. For CFU determinations, $100 \mu \mathrm{l}$ samples were taken before and during antimicrobial challenge on an hourly basis during the first $8 \mathrm{~h}$ and once after $24 \mathrm{~h}$, or after 1,2, 3, 7, 10, 14, and 21 days during long-term experiments. Cells were washed in $1 \% \mathrm{NaCl}$ solution and spotted as $10-\mu \mathrm{l}$ aliquots of serial dilutions onto TSA plates. Colonies were counted after incubation for $24 \mathrm{~h}$ (normally growing colonies) to $48 \mathrm{~h}$ (SCVs) at $37^{\circ} \mathrm{C}$. The lower limit of quantification was $100 \mathrm{CFU} /$ $\mathrm{ml}$. All time-kill experiments were conducted using at least three biological replicates.

\section{Heritability of Persistence}

$2.24 \mathrm{ml}$ of exponential phase cultures were transferred to 14 $\mathrm{ml}$ culture tubes and cells were exposed to 100-fold MIC of tobramycin or 10 -fold MIC of daptomycin for $3 \mathrm{~h}$. Subsequently, cells were washed in $1 \% \mathrm{NaCl}$ solution, resuspended in $16 \mathrm{ml}$ fresh TSB and cultured overnight. Dilution of cells in fresh medium and growth to exponential phase finished the first cycle. Cells were then again subjected to antimicrobial treatment as described and the procedure was conducted in a total of four consecutive cycles.

\section{Acknowledgements}

We thank Tanja Hildebrandt for technical assistance and Friedrich Götz for support. This work was supported by grants BE4038/1 and BE4038/2 within the priority program 1316 'Host Adapted Metabolism of Bacterial Pathogens' and by the research training group Graduate College 685 'Infection Biology: Humanand Plant-Pathogenic Bacteria and Fungi' of the German Research Foundation (DFG), as well as by NIH grant R01 GM061162.

\section{References}

Allison KR, Brynildsen MP, Collins JJ: Heterogeneous bacterial persisters and engineering approaches to eliminate them. Curr Opin Microbiol 2011a;14:593-598.

Allison KR, Brynildsen MP, Collins JJ: Metabolite-enabled eradication of bacterial persisters by aminoglycosides. Nature 2011b;473: 216-220.

Aubry-Damon H, Soussy CJ, Courvalin P: Characterization of mutations in the rpob gene that confer rifampin resistance in Staphylo- coccus aureus. Antimicrob Agents Chemother 1998;42:2590-2594.

Balaban NQ, Merrin J, Chait R, Kowalik L, Leibler S: Bacterial persistence as a phenotypic switch. Science 2004;305:16221625.

- Baltch AL, Ritz WJ, Bopp LH, Michelsen P, Smith RP: Activities of daptomycin and comparative antimicrobials, singly and in combination, against extracellular and intracellular Staphylococcus aureus and its sta- ble small-colony variant in human monocyte-derived macrophages and in broth. Antimicrob Agents Chemother 2008;52: 1829-1833.

- Bates DM, von Eiff C, McNamara PJ, Peters G, Yeaman MR, Bayer AS, Proctor RA: Staphylococcus aureus menD and hemB mutants are as infective as the parent strains, but the menadione biosynthetic mutant persists within the kidney. J Infect Dis 2003;187: 1654-1661. 
Begic D, von Eiff C, Tsuji BT: Daptomycin pharmacodynamics against Staphylococcus aureus hemB mutants displaying the small colony variant phenotype. J Antimicrob Chemother 2009;63:977-981.

- Bigger J: Treatment of staphylococcal infections with penicillin. Lancet 1944;244:497-500.

- Bischoff M, Entenza JM, Giachino P: Influence of a functional sigB operon on the global regulators sar and agr in Staphylococcus aureus. J Bacteriol 2001;183:5171-5179.

- Bradley JJ, Mayhall CG, Dalton HP: Incidence and characteristics of antibiotic-tolerant strains of Staphylococcus aureus. Antimicrob Agents Chemother 1978;13:1052-1057.

-Brooun A, Liu S, Lewis K: A dose-response study of antibiotic resistance in Pseudomonas aeruginosa biofilms. Antimicrob Agents Chemother 2000;44:640-646.

-Chambers HF, Deleo FR: Waves of resistance: Staphylococcus aureus in the antibiotic era. Nat Rev Microbiol 2009;7:629-641.

-Correia FF, D’Onofrio A, Rejtar T, Li L, Karger BL, Makarova K, Koonin EV, Lewis K: Kinase activity of overexpressed hipA is required for growth arrest and multidrug tolerance in Escherichia coli. J Bacteriol 2006; 188:8360-8367.

- Cottarel G, Wierzbowski J: Combination drugs, an emerging option for antibacterial therapy. Trends Biotechnol 2007;25:547-555.

- Dhar N, McKinney JD: Microbial phenotypic heterogeneity and antibiotic tolerance. Curr Opin Microbiol 2007;10:30-38.

-Donegan NP, Cheung AL: Regulation of the mazEF toxin-antitoxin module in Staphylococcus aureus and its impact on sigB expression. J Bacteriol 2009;191:2795-2805.

-Dörr T, Lewis K, Vulic M: SOS response induces persistence to fluoroquinolones in Escherichia coli. PLoS Genet 2009;5:e1000760.

- FuZ, Tamber S, Memmi G, Donegan NP, Cheung AL: Overexpression of $m a z F_{s a}$ in Staphylococcus aureus induces bacteriostasis by selectively targeting $\mathrm{mRNAs}$ for cleavage. J Bacteriol 2009;191:2051-2059.

- Gaupp R, Schlag S, Liebeke M, Lalk M, Götz F: Advantage of upregulation of succinate dehydrogenase in Staphylococcus aureus biofilms. J Bacteriol 2010;192:2385-2394.

-Giachino P, Engelmann S, Bischoff M: Sigma(B) activity depends on rsbU in Staphylococcus aureus. J Bacteriol 2001;183:1843-1852.

-Gunnison JB, Fraher MA, Jawetz E: Persistence of Staphylococcus aureus in penicillin in vitro. J Gen Microbiol 1964;35:335-349.

- Harrison JJ, Turner RJ, Ceri H: Persister cells, the biofilm matrix and tolerance to metal cations in biofilm and planktonic Pseudomonas aeruginosa. Environ Microbiol 2005;7:981994.

Herbert S, Ziebandt AK, Ohlsen K, Schäfer T, Hecker M, Albrecht D, Novick R, Götz F: Repair of global regulators in Staphylococcus aureus 8325 and comparative analysis with other clinical isolates. Infect Immun 2010; 78:2877-2889.
Iordanescu S, Surdeanu M: Two restriction and modification systems in Staphylococcus aureus NCTC8325. J Gen Microbiol 1976;96: 277-281.

Keren I, Kaldalu N, Spoering A, Wang Y, Lewis $\mathrm{K}$ : Persister cells and tolerance to antimicrobials. FEMS Microbiol Lett 2004a;230:13-18.

Keren I, Minami S, Rubin E, Lewis K: Characterization and transcriptome analysis of $\mathrm{Myco}$ bacterium tuberculosis persisters. mBio 2011; 2:e00100-e00111.

Keren I, Shah D, Spoering A, Kaldalu N, Lewis $\mathrm{K}$ : Specialized persister cells and the mechanism of multidrug tolerance in Escherichia coli. J Bacteriol 2004b;186:8172-8180.

Kim JS, Heo P, Yang TJ, Lee KS, Cho DH, Kim BT, Suh JH, Lim HJ, Shin D, Kim SK, Kweon DH: Selective killing of bacterial persisters by a single chemical compound without affecting normal antibiotic-sensitive cells. Antimicrob Agents Chemother 2011;55:53805383.

Lafleur MD, Qi Q, Lewis K: Patients with longterm oral carriage harbor high-persister mutants of Candida albicans. Antimicrob Agents Chemother 2010;54:39-44.

Lewis K: Persister cells, dormancy and infectious disease. Nat Rev Microbiol 2007;5:48-56.

Lewis K: Persister cells. Annu Rev Microbiol 2010a;64:357-372.

Lewis K: Persister cells and the paradox of chronic infections. Microbe 2010b;5:429437.

Mascio CT, Alder JD, Silverman JA: Bactericidal action of daptomycin against stationaryphase and nondividing Staphylococcus aureus cells. Antimicrob Agents Chemother 2007;51:4255-4260.

Mayhall CG, Medoff G, Marr JJ: Variation in the susceptibility of strains of Staphylococcus aureus to oxacillin, cephalothin, and gentamicin. Antimicrob Agents Chemother 1976; 10:707-712.

Möker N, Dean CR, Tao J: Pseudomonas aeruginosa increases formation of multidrug-tolerant persister cells in response to quorumsensing signaling molecules. J Bacteriol 2010;192:1946-1955.

-Moyed HS, Bertrand KP: HipA, a newly recognized gene of Escherichia coli K-12 that affects frequency of persistence after inhibition of murein synthesis. J Bacteriol 1983; 155:768-775.

Moyed HS, Broderick SH: Molecular cloning and expression of hipA, a gene of Escherichia coli K-12 that affects frequency of persistence after inhibition of murein synthesis. J Bacteriol 1986;166:399-403.

Newsom SW: Staphylococcal persisters grown from empyema fluid on L-form medium. J Med Microbiol 1970;3:669-673.

Novick R: Properties of a cryptic high-frequency transducing phage in Staphylococcus aureus. Virology 1967;33:155-166.

- Novick RP, Geisinger E: Quorum sensing in staphylococci. Annu Rev Genet 2008;42: 541-564.
Proctor RA, von Eiff C, Kahl BC, Becker K, McNamara P, Herrmann M, Peters G: Small colony variants: a pathogenic form of bacteria that facilitates persistent and recurrent infections. Nat Rev Microbiol 2006;4:295-305.

- Scherrer R, Moyed HS: Conditional impairment of cell division and altered lethality in hipA mutants of Escherichia coli K-12. J Bacteriol 1988;170:3321-3326.

- Senn MM, Giachino P, Homerova D, Steinhuber A, Strassner J, Kormanec J, Flückiger U, Berger-Bächi B, Bischoff M: Molecular analysis and organization of the $\sigma^{\mathrm{B}}$ operon in Staphylococcus aureus. J Bacteriol 2005;187: 8006-8019.

-Shah D, Zhang Z, Khodursky A, Kaldalu N, Kurg K, Lewis K: Persisters: a distinct physiological state of E. coli. BMC Microbiol 2006;6:53.

-Shapiro JA, Nguyen VL, Chamberlain NR: Evidence for persisters in Staphylococcus epidermidis RP62A planktonic cultures and biofilms. J Med Microbiol 2011;60:950-960.

-Singh R, Ray P, Das A, Sharma M: Role of persisters and small-colony variants in antibiotic resistance of planktonic and biofilm-associated Staphylococcus aureus: an in vitro study. J Med Microbiol 2009;58:1067-1073.

- Spoering AL, Lewis K: Biofilms and planktonic cells of Pseudomonas aeruginosa have similar resistance to killing by antimicrobials. J Bacteriol 2001;183:6746-6751.

- Steenbergen JN, Alder J, Thorne GM, Tally FP: Daptomycin: a lipopeptide antibiotic for the treatment of serious gram-positive infections. J Antimicrob Chemother 2005;55: 283-288.

- Tuchscherr L, Medina E, Hussain M, Völker W, Heitmann V, Niemann S, Holzinger D, Roth J, Proctor RA, Becker K, Peters G, Löffler B: Staphylococcus aureus phenotype switching: an effective bacterial strategy to escape host immune response and establish a chronic infection. EMBO Mol Med 2011;3:129-141.

-Vazquez-Laslop N, Lee H, Neyfakh AA: Increased persistence in Escherichia coli caused by controlled expression of toxins or other unrelated proteins. J Bacteriol 2006; 188: 3494-3497.

Vega NM, Allison KR, Khalil AS, Collins JJ: Signaling-mediated bacterial persister formation. Nat Chem Biol 2012;8:431-433.

von Eiff C: Staphylococcus aureus small colony variants: a challenge to microbiologists and clinicians. Int J Antimicrob Agents 2008;31: 507-510.

von Eiff C, Heilmann C, Proctor RA, Wolz C, Peters G, Götz F: A site-directed Staphylococcus aureus hem $B$ mutant is a small-colony variant which persists intracellularly. J Bacteriol 1997; 179:4706-4712.

-Wiuff C, Zappala RM, Regoes RR, Garner KN, Baquero F, Levin BR: Phenotypic tolerance: antibiotic enrichment of noninherited resistance in bacterial populations. Antimicrob Agents Chemother 2005;49:1483-1494. 